\title{
Gene networks of human hearing impairments: reconstruction and analysis
}

\author{
V.I. Zamyatin ${ }^{1,2 *}$, Z.S. Mustafin ${ }^{1,2}$, E.V. Ignatieva ${ }^{1,2}$, Yu.G. Matushkin ${ }^{1,2}$, \\ O.L. Posukh ${ }^{1,2}$, S.A. Lashin ${ }^{1,2}$ \\ ${ }^{1}$ Institute of Cytology and Genetics SB RAS, Novosibirsk, Russia \\ ${ }^{2}$ Novosibirsk State University, Novosibirsk, Russia \\ *e-mail: zamyatin@bionet.nsc.ru
}

Key words: hearing impairments, gene networks, SNP, transcription factors

\begin{abstract}
Motivation and Aim: Hearing loss is very genetically heterogeneous disorder since more than 100 mapped loci and about one hundred genes are now known to be implicated in hearing impairment (HI). There are nonsyndromic (isolated) forms of hereditary $\mathrm{HI}$ and syndromic HI (combined with other traits/disorders). Despite the fact that a lot of genes are proven to be associated with HIs, there is still no systematic network reconstructions of the molecular and signalling mechanisms of HIs. We tried to reconstruct the gene networks of human HIs considering several layers of biological organization: networks of protein-protein interactions and co-regulation networks.
\end{abstract}

Methods and Algorithms: Sets of genes associated with HIs were extracted from the Hereditary Hearing Loss resource (hereditaryhearingloss.org) as well as from [1]. Transcription factors (TF) from these sets were identified using AnimalTFDB database. Search for the binding sites for all revealed TFs by using Hocomoco MoLoTool (molotool.autosome.ru) with default settings was carried out in the upstream regions $(-2000 ; 0)$ of all genes from these sets. Protein-protein interactions were extracted via GeneMANIA (genemania.org) and String (string-db.org). Network reconstruction was performed in Cytoscape. Evolutionary analysis was made using Orthoscape application [2]. The data on SNPs were extracted from the 1000 Genomes Project and their effects were estimated via Ensembl VEP.

Results: We have reconstructed the gene networks for potential mechanisms of human HIs including gene regulatory and protein-protein interactions networks. The separate regulatory circuits probably corresponding to different HI mechanisms were found in these networks. Moreover, the network analysis revealed several genes encoding TFs which were not previously known to be assosiated with HIs. Evolutionary analysis of the HI gene networks has revealed several "evolutionary young" (TPRN, EDN3, CD164), and also predominant conservation of TFs involved in HIs. Interesting features of the ACTG1 gene were found during the evolutionary analysis and the SNPs functional annotation in human populations: according to $\mathrm{Ka} / \mathrm{Ks}$ ratio, the evolutionary conservation of this gene sequence does not correspond to its SNP enrichness. These findings probably suggest that the influence of stabilizing selection on this gene in human populations has been weakened some time ago. Conclusion: The gene networks including the protein-protein interactions and co-regulation networks were reconstructed for human hearing impairments. The subsequent detailed analysis of these networks will help to expand the set of HI involved genes and understanding of the mechanisms of hearing and its impairments.

Acknowledgements: Partially supported by the RFBR No. 17-29-06016-ofi_m.

\section{References}

1. Stamatiou G., Stankovic K.A. (2013). Comprehensive Network and Pathway Analysis of Human Deafness Genes. Otology Neurotology. 34:961-970.

2. Mustafin Z.S. et al. (2017) Orthoscape: a Cytoscape application for grouping and visualization KEGG based gene networks by taxonomy and homology principles. BMC Bioinformatics. 18(1):S1. 